\title{
Adherence and Persistence with DPP-4 Inhibitors Versus Pioglitazone in Type 2 Diabetes Patients with Chronic Kidney Disease: A Retrospective Claims Database Analysis
}

\author{
Deval Gor, MPharm; Todd A. Lee, PharmD, PhD; Glen T. Schumock, PharmD, MBA, PhD; \\ Surrey M. Walton, MA, PhD; Ben S. Gerber, MPH, MD; Edith A. Nutescu, MS, PharmD; \\ and Daniel R. Touchette, MA, PharmD
}

\begin{abstract}
BACKGROUND: Adherence and persistence with diabetes medication play an important role in glycemic control and may differ by medication class. However, there is a lack of research comparing diabetes medications in patients with renal impairment, despite the challenges and higher burden associated with managing this population.
\end{abstract}

OBJECTIVE: To compare adherence and persistence among patients with type 2 diabetes mellitus (T2DM) and nondialysis chronic kidney disease (CKD) treated with dipeptidyl peptidase-4 (DPP-4) inhibitors versus pioglitazone.

METHODS: This retrospective cohort study used Truven MarketScan administrative claims databases from 2009 to 2015 . One-year adherence for patients with T2DM and nondialysis CKD who initiated therapy with either a DPP-4 inhibitor or pioglitazone was measured by proportion of days covered (PDC) following an initial dispensing, and $P D C \geq 0.80$ was coded as adherent. Persistence was calculated as the days between the index date and last day with the index medication on hand, based on the end of the last days supply or the end of follow-up (i.e., 365 days), whichever occurred first. Multivariate logistic regression and Cox proportional hazards models were used to estimate confounder-adjusted differences between the groups for adherence and persistence.

RESULTS: The final cohort included 9,019 patients (DPP-4 inhibitors: 7,002; pioglitazone: 2,017$)$. In the adjusted analysis, DPP-4 inhibitor users demonstrated a $1.41(95 \% \mathrm{Cl}=1.25-1.59)$ higher odds of being adherent compared with pioglitazone users. Overall adjusted HR for persistence was 0.74 (95\% $\mathrm{Cl}=0.69-0.79$ ), which favored DPP-4 inhibitors compared with pioglitazone. Relative to 2010, persistence with pioglitazone decreased in 2011-2012 and then increased in 2013-2014. In the subgroup analysis, DPP-4 inhibitors first had lower (2010: $\mathrm{OR}=0.78,95 \% \mathrm{Cl}=0.70-0.87 ; 2011-2012$ : $\mathrm{OR}=0.60$, $95 \% \mathrm{Cl}=0.54-0.66)$ and then similar $(2013-2014: 0 \mathrm{R}=1.03,95 \% \mathrm{Cl}=0.88$ 1.19) hazards of nonpersistence compared with pioglitazone.

CONCLUSIONS: Among patients with T2DM and nondialysis CKD, the use of DPP-4 inhibitors was associated with better adherence compared with pioglitazone. However, following the approval of generic pioglitazone and associated lower cost sharing after 2012, the magnitude of difference in adherence between the medication classes reduced. Similarly, safety warnings in 2011 and approval of generic products in 2012 may have affected pioglitazone persistence, leading to first higher and then similar hazards for nonpersistence with pioglitazone as compared with DPP-4 inhibitors. These shifts in the results for pioglitazone warrant further investigation and close monitoring of the population initiating this medication.

J Manag Care Spec Pharm. 2020;26(1):67-75

Copyright $\odot 2020$, Academy of Managed Care Pharmacy. All rights reserved.

\section{What is already known about this subject}

Only selected antidiabetic medications can be prescribed throughout the progression of chronic kidney disease.

Real-world effectiveness of antidiabetic medications depends on adherence in addition to efficacy.

\section{What this study adds}

Among patients with type 2 diabetes and chronic kidney disease, those initiating dipeptidyl peptidase-4 (DPP-4) inhibitors had better adherence compared with those with pioglitazone. Higher cost sharing with DPP-4 inhibitors compared with generic pioglitazone in recent years may undermine this difference. Compared with 2010, persistence with pioglitazone decreased in 2011-2012 following safety warnings and increased in 2013-2014 following approval of the generic

$\mathrm{D}$ iabetes is a highly prevalent chronic metabolic disease that affects $9.4 \%$ of the U.S. population. ${ }^{1}$ Chronic kidney disease (CKD), a common comorbidity and complication of the disease, is estimated to affect approximately $35 \%-40 \%$ of patients with type 2 diabetes mellitus (T2DM). ${ }^{2}$ The risks of cardiovascular complications, hospitalization, and death increase with decreasing kidney function. ${ }^{3}$ In addition, patients with diabetes and CKD have substantially higher medical care costs compared with those patients with diabetes alone. ${ }^{4}$

Achieving glycemic control using pharmacotherapy is essential for the avoidance of disease progression and diabetesrelated complications. Glycemic control has also been associated with better clinical outcomes, including reduced risks of cardiovascular events and progression of CKD and end-stage renal disease (ESRD) among patients with CKD. ${ }^{5}$ Numerous studies have demonstrated the role of adherence in attaining glycemic targets and potential savings for patients with T2DM. ${ }^{6}$ For every $10 \%$ increase in adherence, hemoglobin Alc (HbAlc) decreases by approximately $0.1 \%{ }^{6}$ Nonadherence has been associated with 7\% higher risk of hospitalization, 3.6\% higher mean number of visits, and $10 \%$ higher risk of mortality. ${ }^{6,7}$ Studies have suggested reductions in total medical cost, 
diabetes-related costs, and total annual health care costs with improved adherence by $2 \%, 4 \%$, and $8.5 \%$, respectively. ${ }^{8}$ In addition, adherence to oral diabetes agents is one of the health care quality measures endorsed by the Centers for Medicare $\&$ Medicaid Services. ${ }^{9}$

Oral therapeutic management of diabetes becomes more challenging when patients develop CKD. The National Kidney Foundation Kidney Disease Outcomes Quality Initiative (NKFKDOQI) recommends only selected noninsulin antidiabetic medication classes be used by patients with renal impairment. ${ }^{10}$ Metformin is not recommended with advanced CKD due to risk of lactic acidosis. Further, with progression of diabetes, patients often require treatment intensification with second-line therapy. The major second-line treatment options include sulfonylureas, thiazolidinediones (TZDs), dipeptidyl peptidase-4 (DPP-4) inhibitors, glucagon-like peptide-1 (GLP-1) receptor agonists, and sodium-glucose co-transporter 2 (SGLT-2) inhibitors. GLP-1 agonists and SGLT-2 inhibitors are not recommended in moderate to severe CKD. Although short-acting sulfonylureas are often used, they have a higher risk of hypoglycemia compared with other second-line agents when added to metformin monotherapy and therefore require cautious use by patients with CKD. ${ }^{11}$ The remaining 2 classes, DPP-4 inhibitors and TZDs, are acceptable for use in all stages of $\mathrm{CKD}$, including dialysis.

Meta-analyses of clinical trials have shown that DPP-4 inhibitors and pioglitazone have comparable efficacy as second-line therapies. ${ }^{11,12}$ However, DPP-4 inhibitors exhibit a lower risk of adverse effects, including weight gain, compared with pioglitazone. ${ }^{11}$ While clinical trials provide a comparison between the 2 types of medications, they often exclude patients with renal impairment and do not offer any evidence on utilization patterns and adherence in a real-world clinical setting. In fact, we did not identify any U.S. studies that compared the course of treatment and adherence to these medications in patients with CKD. Furthermore, there were no analyses evaluating the effect of pioglitazone's safety warnings or availability as a generic medication in 2012 on use of these drugs by patients with CKD.

Given the importance of adherence in diabetes management, this study evaluated patient adherence to, persistence with, and treatment patterns of DPP-4 inhibitors and pioglitazone in patients with T2DM and CKD.

\section{Methods}

\section{Data Source}

This study used a retrospective cohort design of administrative claims data from Truven Health MarketScan Commercial and Medicare Supplemental databases from January 2009 through September 2015. These databases capture person-specific enrollment, health care utilization, and expenditures from over 350 payers comprising large employers, health plans, and government and public organizations. The University of Illinois at Chicago Office for the Protection of Research Subjects determined that the pharmaceutical outcomes research using these databases does not meet the definition of human subject research as defined by 45 CFR 46.102(f).

\section{Cohort Selection}

Patients with T2DM and nondialysis CKD who initiated therapy with either a DPP-4 inhibitor or pioglitazone were selected using 2 steps. First, enrollees with at least 1 prescription claim for a DPP-4 inhibitor or pioglitazone were identified. Second, enrollees with at least 1 inpatient or 1 outpatient claim (at least 1 day apart from each other) for each of diabetes and CKD were identified using International Classification of Diseases, Ninth Revision, Clinical Modification (ICD-9-CM) diagnosis codes (see Appendix A, available in online article). The first prescription claim date for either of the study medications was determined to be the index date, and the respective type of medication (i.e., DPP-4 inhibitor or pioglitazone) was the index medication. The study included only new users, defined as initiating either a DPP-4 inhibitor or pioglitazone with absence of prescription claims for both DPP-4 inhibitors and TZDs within a year before the index date. Patients were required to have at least 1 ICD-9-CM diagnosis code for diabetes and CKD within a year before the index date. Other inclusion criteria were age $>30$ years at the index date, absence of claims for gestational diabetes during the study period, and continuous enrollment for 1 year before and after the index date.

Patients with contraindications to the index medication or with disease conditions that might restrict the use of the index medication, based on recent safety warnings from the U.S. Food and Drug Administration (FDA), were excluded. This included all patients with inpatient or outpatient ICD-9-CM codes indicating the presence of bladder cancer, pancreatic cancer, or heart failure. Patients with diagnosis and procedural codes for ESRD, dialysis, or kidney transplant were also excluded from the study.

Information on patient demographics, health care plan characteristics, index medication days supply, and index medication cost sharing for 30 days supply were identified at the index date. Periods of 90 days before and after the index date were used to identify use of non-index concurrent diabetes medications. Data on comorbid conditions, diabetes and total health care resource use, diabetes and total health care cost, previous diabetes medications, and other nondiabetes medications were extracted for the 12 -month period preceding the index date.

\section{Outcomes}

The primary outcomes of this study were medication adherence and persistence with DPP-4 inhibitors or pioglitazone in the 12 months following the index date. Medication adherence was 


\section{Adherence and Persistence with DPP-4 Inhibitors Versus Pioglitazone in Type 2 Diabetes Patients with Chronic Kidney Disease: A Retrospective Claims Database Analysis}

measured for patients with at least 2 prescription fills using proportion of days covered (PDC). PDC was calculated using the sum of days supply from the index date through the last dispensing divided by the total days of the follow-up period (i.e., 365 days). ${ }^{13}$ Availability of medication was determined for each day of the follow-up period based on the days supply of the dispensed prescriptions. If a patient refilled a medication before the end of the supply for the previous dispensing, the start date of the subsequent dispensing was adjusted to the end of the supply from the previous dispensing. The end of the last days supply was capped at 365 days. Switching within the index medication class was allowed. If a patient switched from the medication class of the index medication to a new medication class that was not part of the diabetes regimen at the index date, then the denominator and numerator were censored at the date of switch for calculating the PDC for the index medication. Adherence to study medications was also compared at 4 levels of cost sharing based on its median and quartiles.

Persistence was calculated as the days between the index date and last day with the index medication on hand, based on the end of the last days supply or the end of follow-up (i.e., 365 days), whichever occurred first. ${ }^{13}$ A permissible gap of 2 times the days supply without index medication was allowed in between the prescriptions before classifying an individual as nonpersistent. Patients with continuous medication use for 365 days, allowing for permissible gaps, were considered persistent. As part of a sensitivity analysis, persistence was also calculated using permissible gaps of 60 days and 90 days. Further, subgroup analyses were carried out for 3 calendar time periods (2010, 2011-2012, and 2013-2014) to evaluate the effect of FDA warnings and availability of generic pioglitazone on persistence.

In addition to these primary outcomes, treatment patterns were also observed during follow-up. Diabetes medication regimens were defined at the medication class-level for medications where there was (a) at least 1 prescription claim within 60 days of index date or (b) at least 1 prescription claim in each time window of 90 days before and after the index date. For assessing treatment patterns, the patients were categorized into 4 mutually exclusive groups based on the changes in treatment regimen during the follow-up period: (1) patients staying on the current regimen with no changes; (2) patients requiring intensification, defined as addition of a new antidiabetic class that was not part of the current regimen, with at least 60 days of overlap with the index medication class; (3) patients requiring switching, defined as discontinuation of index medication class (DPP-4 inhibitor or pioglitazone) and initiation of a new antidiabetic class before the end of days supply of index class plus 60 days; and (4) patients discontinuing index medication class without initiation of a new antidiabetic class.

\section{FIGURE 1 Study Selection Process}

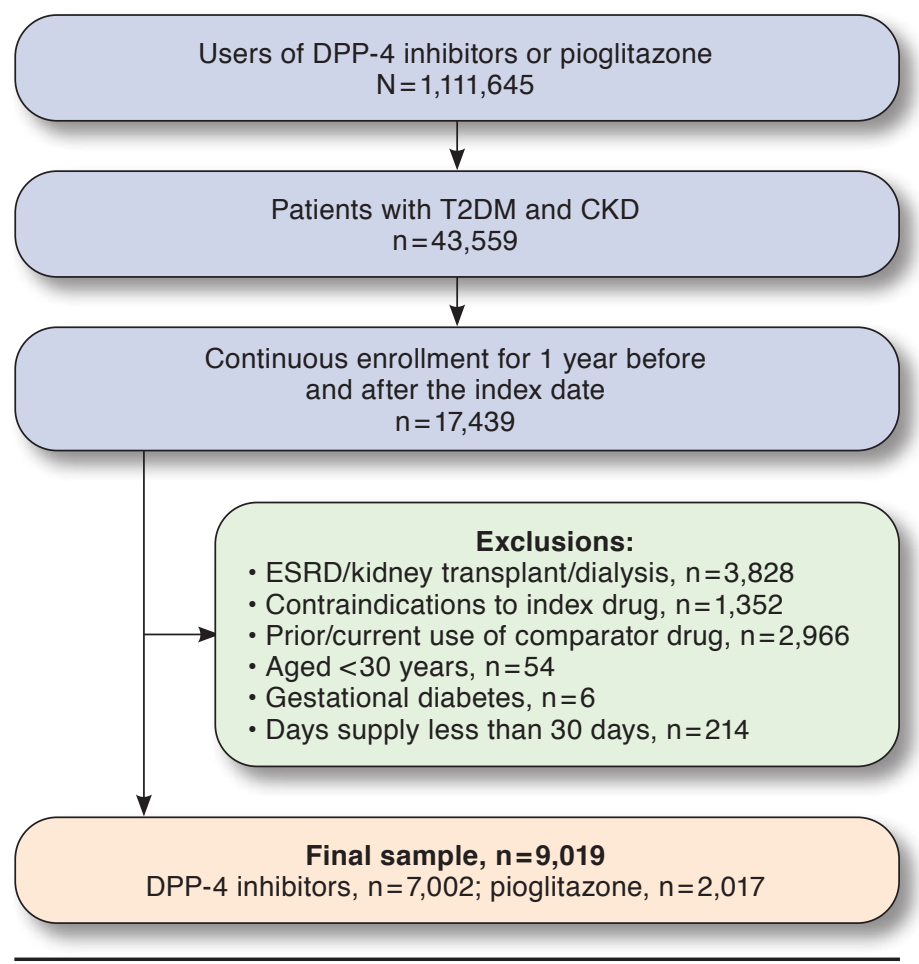

$C K D=$ chronic kidney disease $; D P P-4=$ dipeptidyl peptidase-4; ESRD =end-stage renal disease; $T 2 D M=$ type 2 diabetes mellitus.

\section{Statistical Analysis}

Descriptive analyses were carried out to summarize the baseline characteristics of patients initiating DPP-4 inhibitors and pioglitazone. In a bivariate analysis, differences between the 2 cohorts were evaluated using t-tests for normally distributed continuous variables, Wilcoxon rank sum tests for continuous variables with non-normal distributions, and chi-square tests for categorical variables.

The mean PDC and dichotomous persistence were compared between the 2 groups using the Wilcoxon rank sum test and chi-square test, respectively. Patients were categorized as adherent if their PDCs were $\geq 0.80$. In a bivariate analysis, baseline variables associated with adherence and index medication were identified as covariates. These variables were considered for inclusion in the multivariable logistic regression model with PDC as a dichotomous dependent variable and index medication as the independent variable. A final multivariate model was selected based on the use of a manual backward elimination process that included removing nonsignificant $(P>0.05)$ variables until each covariate remained statistically significant. Using a similar model selection process, a Cox proportional hazards model adjusted for covariates was developed to estimate hazard ratios (HRs) for nonpersistence. 


\section{Adherence and Persistence with DPP-4 Inhibitors Versus Pioglitazone in Type 2 Diabetes Patients with Chronic Kidney Disease: A Retrospective Claims Database Analysis}

\section{TABLE 1$)$ Baseline Characteristics}

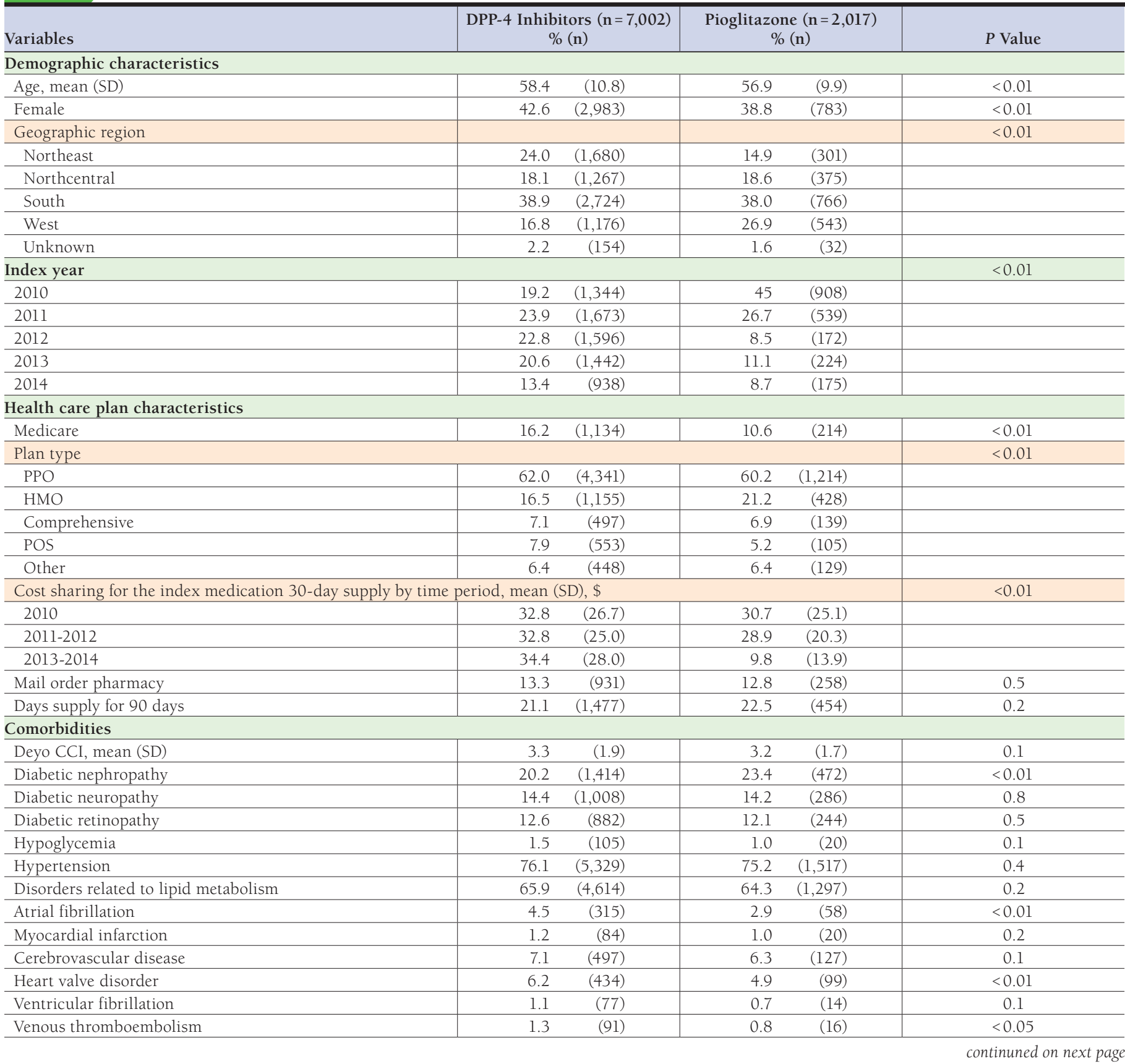

Also, an interaction term for calendar time period was added to assess the difference in persistence between the medications over the years 2010, 2011-2012, and 2013-2014.

Treatment patterns were described using the proportion of patients falling into each of the 4 categories. No change, intensification, switching, and discontinuation were compared using chi-square tests for each time period. In addition, multinomial logistic regression adjusting for covariates was used to compare odds of intensification, switching, and discontinuation as compared with staying on the index medication with no change between DPP-4 inhibitors and pioglitazone. For all statistical analyses, two-tailed significance was assessed at an alpha of 0.05. All analyses were conducted using SAS version 9.4 (SAS Institute, Cary, NC). 


\section{Adherence and Persistence with DPP-4 Inhibitors Versus Pioglitazone in Type 2 Diabetes Patients with Chronic Kidney Disease: A Retrospective Claims Database Analysis}

TABLE 1 Baseline Characteristics (continued)

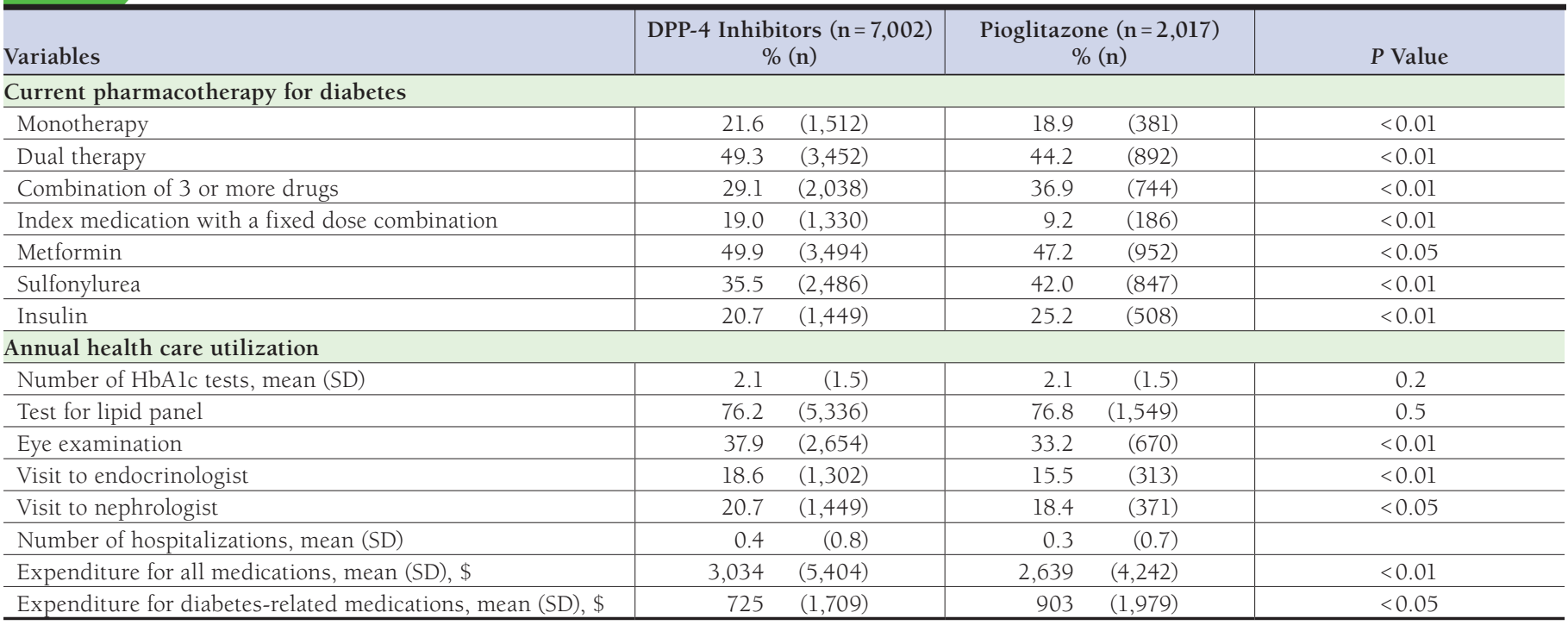

$C C I=$ Charlson Comorbidity Index; DPP-4 = dipeptidyl peptidase-4; HbAlc=hemoglobin Alc; HMO=health maintenance organization; PPO= preferred provider organization; $P O S=$ point of service; $S D=$ standard deviation

\section{Results}

As shown in Figure 1, a cohort of 9,019 patients was identified after application of the inclusion and exclusion criteria. A greater number of patients were using DPP-4 inhibitors $(\mathrm{n}=7,002)$ than pioglitazone $(\mathrm{n}=2,017)$. The demographics and baseline characteristics for the 2 groups are shown in Table 1 . In general, patients who initiated DPP-4 inhibitors were slightly older, more likely to be female, and had higher cardiovascular comorbidities.

The mean PDC (mean \pm SD: $0.77 \pm 0.25$ vs. $0.72 \pm 0.27$, $P<0.01)$ and proportion of patients with $P D C \geq 0.80$ (59.5\% vs. $52.4 \%, P<0.01)$ were higher for patients taking DPP-4 inhibitors (Table 2). Patients initiating DPP-4 inhibitors had higher odds of being adherent than those who initiated pioglitazone (adjusted odds ratio $[\mathrm{AOR}]=1.41,95 \%$ confidence interval $[C I]=1.25-1.59$ ). In a stratified analysis by cost sharing at each level, patients with DPP-4 inhibitors were more likely to be considered adherent than patients taking pioglitazone $(P<0.01)$. Restricting the analysis to a time period when generic pioglitazone was available (2013-2014) and removing cost sharing as a covariate from the regression model, a smaller difference in adherence was observed that failed to reach statistical significance (AOR $=1.18,95 \% \mathrm{CI}=0.92-1.51$; Table 2).

A higher proportion of patients on DPP-4 inhibitors persisted for 1 year compared with patients on pioglitazone (56.7\% vs. $46.3 \%, P<0.01)$. In the sensitivity analyses, different definitions for allowed gaps between refills changed the proportion defined as persistent but had no substantial effect on the magnitude of difference between the groups. The Kaplan-
Meier survival curve for time to nonpersistence is shown in Figure 2. Days on therapy for pioglitazone varied across the years, and the interaction term between the index medication and time period was significant $(P<0.01)$ in the multivariate Cox proportional hazards model. In this adjusted time to event analysis, the overall adjusted $\mathrm{HR}$ was 0.74 (95\% CI =0.69-0.79). In a subgroup analysis by time period, the estimates for HR were 0.78 (95\% CI $=0.70-0.87), 0.60(95 \% \mathrm{CI}=0.54-0.66)$, and 1.03 (95\% CI =0.88-1.19) in 2010, 2011-2012, and 2013-2014, respectively (Table 2). Changes in adherence and persistence by quarters over the study time period are described in Appendix B (available in online article).

Treatment patterns for 1 year following the index date are shown in Table 2. Significant differences were observed in treatment patterns for the time periods 2010 and 2011-2012 $(P<0.01)$. However, for the index years 2013-2014, no significant differences were observed in treatment patterns between the 2 groups $(P=0.14)$. In the multivariate adjusted analysis, DPP-4 inhibitors were associated with lower odds of switching (2010 $\mathrm{AOR}=0.60,95 \% \mathrm{CI}=0.44-0.82 ; 2011-2012 \quad \mathrm{AOR}=0.31,95 \%$ $\mathrm{CI}=0.23-0.40)$ and discontinuation (2010 $\mathrm{AOR}=0.72,95 \%$ $\mathrm{CI}=0.58-0.89 ; 2011-2012 \mathrm{AOR}=0.46,95 \% \mathrm{CI}=0.37-0.56) \mathrm{com}-$ pared with pioglitazone from 2010-2012. In 2013-2014, although DPP-4 inhibitors had lower odds of switching and discontinuation, the difference was not statistically significant (Table 2)

\section{Discussion}

In this retrospective analysis of pharmacy claims for patients with T2DM and nondialysis CKD, those using DPP-4 inhibitors 


\section{Adherence and Persistence with DPP-4 Inhibitors Versus Pioglitazone in Type 2 Diabetes Patients with Chronic Kidney Disease: A Retrospective Claims Database Analysis}

\section{TABLE 2 Results for Primary and Secondary Outcomes}

\begin{tabular}{|c|c|c|c|c|c|c|}
\hline Outcome Type & \multicolumn{2}{|c|}{ DPP-4 Inhibitors } & \multicolumn{2}{|c|}{ Pioglitazone } & $P$ Value & Adjusted Results \\
\hline Adherence & \multicolumn{2}{|c|}{$\mathrm{n}=5,946$} & \multicolumn{2}{|c|}{$\mathrm{n}=1,618$} & & Adjusted OR $(95 \% \mathrm{CI})$ \\
\hline PDC, mean (SD) & 0.77 & $(0.25)$ & 0.72 & $(0.27)$ & $<0.01$ & \\
\hline PDC, median (IQR) & 0.88 & $(0.62-0.98)$ & 0.82 & $(0.49-0.98)$ & $<0.01$ & \\
\hline Adherence (PDC $\geq 0.8$ ) for full cohort, $\%(n)$ & 59.5 & $(3,538)$ & 52.4 & $(848)$ & $<0.01$ & $1.41 \quad(1.25-1.59)^{\mathrm{a}}$ \\
\hline \multicolumn{7}{|c|}{ Adherence (PDC $\geq 0.8$ ) by cost sharing levels, $\%(n)$} \\
\hline$\$ 0-\$ 15$ & 65.3 & $(3,883)$ & 57.9 & $(937)$ & $<0.01$ & \\
\hline$\$ 16-\$ 30$ & 61.6 & $(3,663)$ & 53.2 & $(861)$ & $<0.01$ & \\
\hline$\$ 31-\$ 45$ & 58.0 & $(3,449)$ & 48.5 & $(785)$ & $<0.01$ & \\
\hline$>\$ 45$ & 53.5 & $(3,181)$ & 42.2 & $(683)$ & $<0.01$ & \\
\hline \multicolumn{7}{|l|}{ Adherence (PDC $\geq 0.8$ ) by index years, $\%(n)$} \\
\hline 2010 & 61.1 & $(3,633)$ & 53.0 & $(858)$ & & $1.47 \quad(1.17-1.83)^{\mathrm{b}}$ \\
\hline 2011-2012 & 59.3 & $(3,526)$ & 48.3 & $(781)$ & & $1.60 \quad(1.20-1.96)^{\mathrm{b}}$ \\
\hline 2013-2014 & 58.8 & $(3,496)$ & 57.4 & $(929)$ & & $1.18 \quad(0.92-1.51)^{\mathrm{b}}$ \\
\hline Persistence & \multicolumn{2}{|c|}{$\mathbf{n}=7,002$} & \multicolumn{2}{|c|}{$\mathrm{n}=2,017$} & & Adjusted HR $(95 \% \mathrm{CI})^{\mathrm{c}}$ \\
\hline \multicolumn{7}{|l|}{ Persistence for full cohort, \% (n) } \\
\hline Allowed gap: 2 times days supply & 56.7 & $(3,970)$ & 46.3 & $(934)$ & $<0.01$ & $0.74 \quad(0.69-0.79)$ \\
\hline Allowed gap: 60 days & 50.2 & $(3,515)$ & 39.7 & $(801)$ & $<0.01$ & \\
\hline Allowed gap: 90 days & 58.2 & $(4,075)$ & 47.6 & $(960)$ & $<0.01$ & \\
\hline \multicolumn{7}{|l|}{ Days on therapy by index years, median (IQR) } \\
\hline 2010 & 355 & $(91-365)$ & 260 & $(88-365)$ & & $0.78 \quad(0.70-0.87)$ \\
\hline 2011-2012 & 351 & $(96-365)$ & 168 & $(73-365)$ & & $0.60 \quad(0.54-0.66)$ \\
\hline 2013-2014 & 339 & $(91-365)$ & 349 & $(118-365)$ & & $1.03 \quad(0.88-1.19)$ \\
\hline Treatment patterns, \% (n) & \multicolumn{2}{|c|}{$\mathbf{n}=7,002$} & \multicolumn{2}{|c|}{$\mathrm{n}=2,017$} & & Adjusted OR, $(95 \% \mathrm{CI})^{\mathrm{d}}$ \\
\hline 2010 & & & & & $<0.01$ & \\
\hline No change & 47.9 & $(3,354)$ & 39.3 & $(793)$ & & Reference \\
\hline Intensification & 13.8 & $(966)$ & 11.0 & $(222)$ & & $1.01 \quad(0.75-1.36)$ \\
\hline Switching & 9.0 & $(630)$ & 13.3 & (268) & & $0.60 \quad(0.44-0.82)$ \\
\hline Discontinuation & 29.3 & $(2,052)$ & 36.3 & $(732)$ & & $0.72 \quad(0.58-0.89)$ \\
\hline $2011-2012$ & & & & & $<0.01$ & \\
\hline No change & 48.4 & $(3,389)$ & 30.1 & $(607)$ & & Reference \\
\hline Intensification & 11.7 & $(819)$ & 12.0 & $(242)$ & & $0.55 \quad(0.41-0.74)$ \\
\hline Switching & 8.9 & $(623)$ & 16.9 & $(341)$ & & $0.31 \quad(0.23-0.40)$ \\
\hline Discontinuation & 31.0 & $(2,171)$ & 41.0 & $(827)$ & & $0.46 \quad(0.37-0.56)$ \\
\hline $2013-2014$ & & & & & 0.14 & \\
\hline No change & 45.6 & $(3,193)$ & 44.1 & (889) & & Reference \\
\hline Intensification & 13.7 & $(959)$ & 18.1 & $(365)$ & & $0.66 \quad(0.47-0.91)$ \\
\hline Switching & 10.3 & $(721)$ & 9.5 & (192) & & $0.87 \quad(0.58-1.31)$ \\
\hline Discontinuation & 30.4 & $(2,129)$ & 28.3 & $(571)$ & & $0.87 \quad(0.66-1.15)$ \\
\hline \multicolumn{7}{|c|}{ 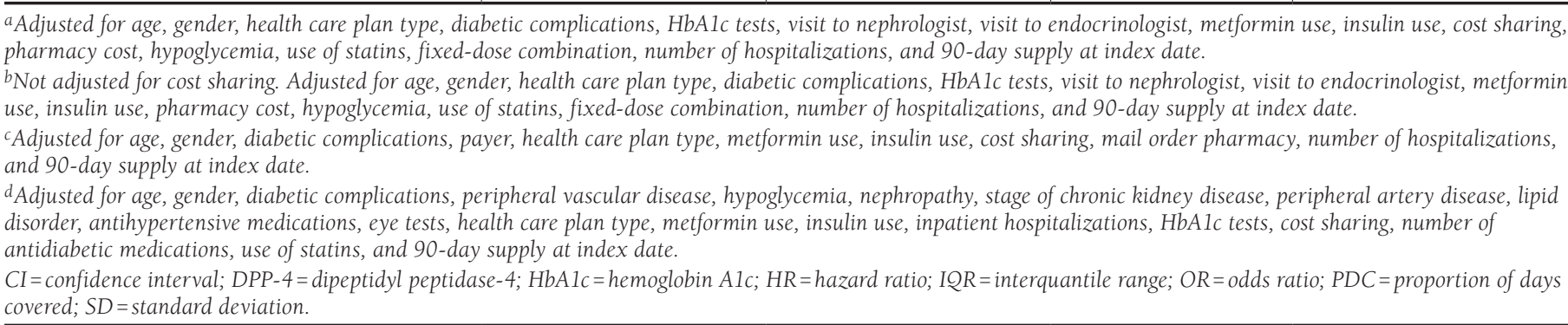 } \\
\hline
\end{tabular}

had moderately higher adherence compared with those who used pioglitazone. The DPP-4 inhibitors users also had longer persistence for the years 2010-2012. However, following the approval of generic pioglitazone, no significant difference in persistence was observed between the 2 medications.
Patients with comorbid CKD represent a subgroup with worse disease progression and higher health care resource use, so adherence to medications needs to be monitored particularly closely. ${ }^{4}$ DPP-4 inhibitors and pioglitazone are acceptable second-line therapies in patients with CKD. Despite similar 


\section{FIGURE 2 Survival Curve for Time to Nonpersistence in Days for Patients Using DPP-4 Inhibitors}

or Pioglitazone

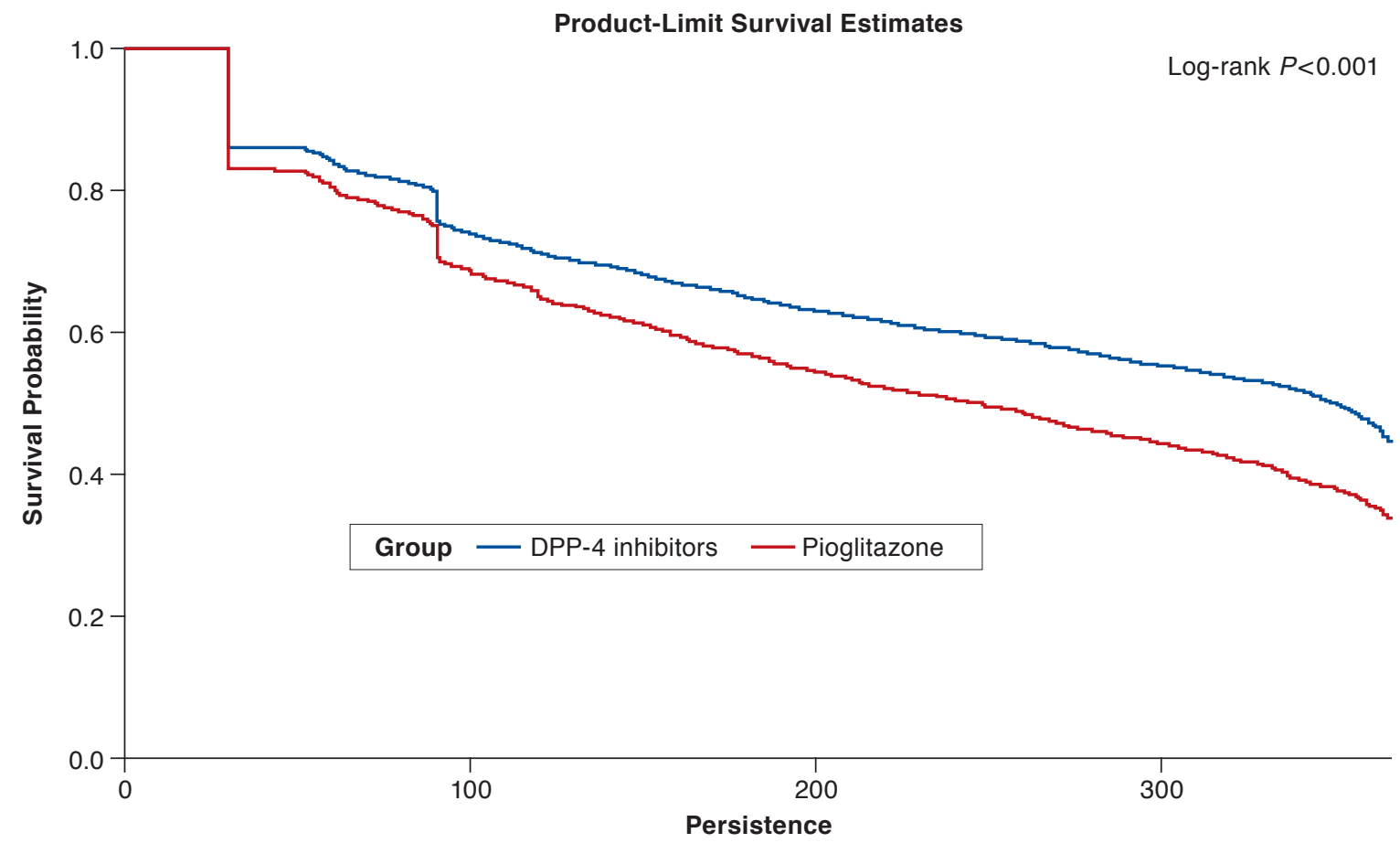

DPP-4 = dipeptidyl peptidase -4 .

efficacy in reducing HbAlc in clinical trials, effectiveness in practice depends on the adherence to these medications. ${ }^{14}$ Lower adherence with pioglitazone observed in our study may contribute towards reduced effectiveness and poorer diabetes outcomes, including progression of CKD. ${ }^{15}$ Poor adherence also has been associated with intensification of therapy, increased diabetes distress, and reduced diabetes-related quality of life. ${ }^{16-18}$ Results of our study can help providers and health policymakers make informed decisions about therapy selection and ensure better diabetes management for the patients with concerns of nonadherence. Apart from clinical benefits for patients, higher adherence rates can ensure better star ratings and may provide financial incentives for health care systems and providers.

Similar to our study, previous studies using data before 2012 in the general diabetic population have observed better adherence (AOR $=1.60,95 \% \mathrm{CI}=1.56-1.65)$ with DPP-4 inhibitors compared with TZDs. ${ }^{19,20}$ The difference in the magnitude of odds ratios compared with our study could be explained by differences in study populations, study time periods (i.e., before vs. after pioglitazone generic approval), and the method used for PDC calculation, in which we censored the patients when they switched the index medication. In contrast to other studies, Flory et al. (2017) observed a lower adherence rate with DPP-4 inhibitors compared with TZDs (daily medication possession probability: 0.36 vs. 0.21 ). ${ }^{21}$ One possible explanation could be greater differences in cost sharing between medications, which were not controlled for in their analysis.

In general, cost sharing has been found to be inversely associated with adherence. ${ }^{22,23}$ In our stratified analysis, we observed similar trends. For both groups, the mean PDC decreased with an increase in cost sharing. Among patients with the index year during 2013-2014, cost-related nonadherence may have decreased for generic pioglitazone, leading to an attenuated odds ratio for adherence compared with DPP-4 inhibitors. The higher cost sharing for DPP-4 inhibitors can undermine its effectiveness among patients whose adherence is sensitive to drug cost. Health care plans should be tailored to optimize out-of-pocket costs for patients, since high medication cost was the most common reason $(21.3 \%)$ for nonadherence in a survey of patients with CKD. ${ }^{24}$

Persistence with pioglitazone changed dramatically over time. The FDA initiated a safety review for pioglitazone and risk of bladder cancer in September 2010. ${ }^{25}$ In June 2011, the FDA issued a safety warning for pioglitazone, recognizing a possible increased risk of bladder cancer after exposure of more than 


\section{Adherence and Persistence with DPP-4 Inhibitors Versus Pioglitazone in Type 2 Diabetes Patients with Chronic Kidney Disease: A Retrospective Claims Database Analysis}

1 year based on an interim analysis of a 10 -year observational study. ${ }^{26,27}$ The FDA also updated the label to suggest that health care professionals not use pioglitazone for patients with active bladder cancer, to use caution when patients have a history of bladder cancer, and to monitor patients taking pioglitazone for development of any signs of this disease. This may explain the lower persistence levels for the years 2011-2012 compared with 2010 and the relatively higher rates of switching and discontinuation as shown in treatment patterns. In late 2012, the first generic pioglitazone was approved. This likely affected cost sharing and tier placement in the formulary, leading to improvement in adherence and persistence (Appendix B).

\section{Limitations}

Given the nature of the data and methods used, this study had several limitations. First, medication adherence was assessed based on the pharmacy claims data, which may not correlate accurately with how patients actually took the medication. Second, the administrative claims database did not provide socioeconomic or clinical variables such as race, income, education, $\mathrm{HbAlc}$, duration of diabetes, and glomerular filtration rate, with potential for residual confounding. Third, a claims-based diagnosis of CKD has relatively lower sensitivity, which limits the generalizability of results beyond this selective population. The results are applicable to nondialysis patients; however, the possibility for misidentification of the population cannot be neglected if the patients received dialysis but the claims were absent in the database. Fourth, determining the reasons for nonadherence, along with the long-term effect of adherence on clinical outcomes, were beyond the scope of this study. Finally, the study population comprised primarily commercially insured patients, which may differ from those with Medicare alone, Medicaid, or no insurance.

\section{Conclusions}

Patients with T2DM and nondialysis CKD who initiated DPP-4 inhibitors adhered more to their medications compared with those initiating pioglitazone. The difference between adherence decreased following introduction of low-cost generic pioglitazone, suggesting that cost sharing may be an important factor in determining adherence to these medications. This finding can help providers make an informed decision when selecting therapy for patients with renal impairment.

The duration of therapy changed substantially among the pioglitazone initiators, which coincided with the timeline of the FDA warnings and its generic approval. This shift in the results, and the attitudes towards the changing status of pioglitazone warrants further investigation. Nonetheless, pharmacotherapy-related decisions should be based on multiple factors that include patient characteristics, contraindications to therapy, and intended outcomes, in addition to the formulary status or cost of diabetes medications.

\section{Authors}

DEVAL GOR, MPharm, Department of Pharmacy Systems, Outcomes and Policy, College of Pharmacy, University of Illinois at Chicago. TODD A. LEE, PharmD, PhD; GLEN T. SCHUMOCK, PharmD, MBA, PhD; SURREY M. WALTON, MA, PhD; EDITH A. NUTESCU, MS, PharmD; and DANIEL R. TOUCHETTE, MA, PharmD, Department of Pharmacy Systems, Outcomes and Policy, and Center for Pharmacoepidemiology and Pharmacoeconomic Research, College of Pharmacy, University of Illinois at Chicago. BEN S. GERBER, MPH, MD, Division of Academic Internal Medicine and Geriatrics, College of Medicine, University of Illinois at Chicago.

AUTHOR CORRESPONDENCE: Daniel R. Touchette, MA, PharmD, University of Illinois at Chicago, 833 S. Wood St., Rm 256 (M/C 871), Chicago, IL 60612. Tel.: 312.355.3204; E-mail: drtouche@uic.edu.

\section{DISCLOSURES}

No funding was received for this study. The authors have no conflicts of interest to disclose.

An abstract for this study was presented as a podium presentation at the International Society of Pharmacoeconomics and Outcomes Research (ISPOR) 2019 Annual Meeting; May 18-22, 2019; New Orleans, LA

\section{REFERENCES}

1. Centers for Disease Control and Prevention. National diabetes statistics report, 2017. Available at: https:/www.cdc.gov/diabetes/pdfs/data/statistics/ national-diabetes-statistics-report.pdf. Accessed November 18, 2019.

2. Murphy D, McCulloch CE, Lin F, et al. Trends in prevalence of chronic kidney disease in the United States. Ann Intern Med. 2016;165(7):473-81.

3. Go AS, Chertow GM, Fan D, McCulloch CE, Hsu CY. Chronic kidney disease and the risks of death, cardiovascular events, and hospitalization. N Engl J Med. 2004;351(13):1296-305.

4. Vupputuri S, Kimes TM, Calloway MO, et al. The economic burden of progressive chronic kidney disease among patients with type 2 diabetes. J Diabetes Complications. 2014;28(1):10-16.

5. Shurraw S, Hemmelgarn B, Lin M, et al. Association between glycemic control and adverse outcomes in people with diabetes mellitus and chronic kidney disease: a population-based cohort study. Arch Intern Med. 2011;171(21):1920-27.

6. Asche C, LaFleur J, Conner C. A review of diabetes treatment adherence and the association with clinical and economic outcomes. Clin Ther 2011;33(1):74-109

7. Huber CA, Rapold R, Brungger B, Reich O, Rosemann T. One-year adherence to oral antihyperglycemic medication and risk prediction of patient outcomes for adults with diabetes mellitus: an observational study. Medicine (Baltimore). 2016;95(26):e3994.

8. Shenolikar RA, Balkrishnan R, Camacho FT, Whitmire JT, Anderson RT Comparison of medication adherence and associated health care costs after introduction of pioglitazone treatment in African Americans versus all other races in patients with type 2 diabetes mellitus: a retrospective data analysis. Clin Ther. 2006;28(8):1199-207.

9. Centers for Medicare \& Medicaid Services. Quality measures. Retrieved December 2017. Available at: https://www.cms.gov/Medicare/QualityInitiatives-Patient-Assessment-Instruments/QualityMeasures/index.html. Accessed November 18, 2019. 


\section{Adherence and Persistence with DPP-4 Inhibitors Versus Pioglitazone in Type 2 Diabetes Patients with Chronic Kidney Disease: A Retrospective Claims Database Analysis}

10. National Kidney Foundation. KDOQI clinical practice guideline for diabetes and CKD: 2012 update. Am J Kidney Dis. 2012;60(5):850-86.

11. Karagiannis T, Paschos P, Paletas K, Matthews DR, Tsapas A. Dipeptidyl peptidase- 4 inhibitors for treatment of type 2 diabetes mellitus in the clinical setting: systematic review and meta-analysis. BMJ. 2012;344:e1369.

12. McIntosh B, Cameron C, Singh SR, et al. Second-line therapy in patients with type 2 diabetes inadequately controlled with metformin monotherapy: a systematic review and mixed-treatment comparison meta-analysis. Open Med. 2011;5(1):e35-48.

13. Peterson AM, Nau DP, Cramer JA, Benner J, Gwadry-Sridhar F, Nichol M. A checklist for medication compliance and persistence studies using retrospective databases. Value Health. 2007;10(1):3-12.

14. Gordon J, McEwan P, Idris I, Evans M, Puelles J. Treatment choice, medication adherence and glycemic efficacy in people with type 2 diabetes: a UK clinical practice database study. BMJ Open Diabetes Res Care. 2018;6(1):e000512.

15. Cedillo-Couvert EA, Ricardo AC, Chen J, et al. Self-reported medication adherence and CKD progression. Kidney Int Rep. 2018;3(3):645-51.

16. Jannoo Z, Wah YB, Lazim AM, Hassali MA. Examining diabetes distress, medication adherence, diabetes self-care activities, diabetes-specific quality of life and health-related quality of life among type 2 diabetes mellitus patients. J Clin Transl Endocrinol. 2017;9:48-54

17. Alfian SD, Sukandar H, Lestari K, Abdulah R. Medication adherence contributes to an improved quality of life in type 2 diabetes mellitus patients: a cross-sectional study. Diabetes Ther. 2016;7(4):755-64.

18. Kogut SJ, Andrade SE, Willey C, Larrat EP. Nonadherence as a predictor of antidiabetic drug therapy intensification (augmentation), Pharmacoepidemiol Drug Saf. 2004;13(9):591-98.
19. Curkendall SM, Thomas N, Bell KF, Juneau PL, Weiss AJ. Predictors of medication adherence in patients with type 2 diabetes mellitus. Curr Med Res Opin. 2013;29(10):1275-86.

20. Farr AM, Sheehan JJ, Curkendall SM, Smith DM, Johnston SS, Kalsekar I. Retrospective analysis of long-term adherence to and persistence with DPP-4 inhibitors in US adults with type 2 diabetes mellitus. Adv Ther. 2014;31(12):1287-305.

21. Flory J, Gerhard T, Stempniewicz N, Keating S, Rowan CG. Comparative adherence to diabetes drugs: an analysis of electronic health records and claims data. Diabetes Obes Metab. 2017;19(8):1184-87.

22. Gibson TB, Song X, Alemayehu B, et al. Cost sharing, adherence, and health outcomes in patients with diabetes. Am J Manag Care. 2010;16(8):589-600.

23. Kirkman MS, Rowan-Martin MT, Levin R, et al. Determinants of adherence to diabetes medications: findings from a large pharmacy claims database. Diabetes Care. 2015;38(4):604-09.

24. Sontakke S, Budania R, Bajait C, Jaiswal K, Pimpalkhute S. Evaluation of adherence to therapy in patients of chronic kidney disease. Indian J Pharmacol. 2015:47(6):668-71.

25. U.S. Food and Drug Administration. FDA reviewing preliminary safety information on Actos (pioglitazone). Press release. September 17, 2010. Available at: https://wayback.archive-it.org/7993/20170112032006/ http:/www.fda.gov/NewsEvents/Newsroom/PressAnnouncements/2010/ ucm226244.htm. Accessed November 18, 2019.

26. U.S. Food and Drug Administration. FDA drug safety communication: update to ongoing safety review of Actos (pioglitazone) and increased risk of bladder cancer. June 15, 2011. Available at: https://www.fda.gov/Drugs/ DrugSafety/ucm259150.htm. Accessed November 18, 2019.

27. Lewis JD, Ferrara A, Peng T, et al. Risk of bladder cancer among diabetic patients treated with pioglitazone: interim report of a longitudinal cohort study. Diabetes Care. 2011;34(4):916-22. 
Adherence and Persistence with DPP-4 Inhibitors Versus Pioglitazone in Type 2 Diabetes

Patients with Chronic Kidney Disease: A Retrospective Claims Database Analysis

\begin{tabular}{|c|c|}
\hline Condition & Codes \\
\hline Diabetes & ICD-9-CM diagnosis codes: $250 . \mathrm{xx}$ \\
\hline CKD & $\begin{array}{l}\text { ICD-9 diagnosis codes: } 250.4 \mathrm{x}, 274.10,403 . \mathrm{xx} \\
\text { 404.xx, 440.1x, 442.1x, 453.3x, 581.xx, 582.xx, } \\
\text { 583.xx, 585.xx, 586.xx, 587.xx, 593.xx, 753.0x, 753.3x, 866.00, 866.01, 866.1x }\end{array}$ \\
\hline Gestational diabetes & ICD-9-CM diagnosis codes: $648.0,648.8$ \\
\hline Bladder cancer & ICD-9-CM diagnosis codes: $233.7,188 . x, \mathrm{~V} 10.51$ \\
\hline Pancreatic cancer & ICD-9-CM diagnosis codes: $157 . \mathrm{x}$ \\
\hline Heart failure & ICD-9-CM diagnosis codes: 428.x, 398.91 \\
\hline ESRD & ICD-9-CM diagnosis code: 585.6 \\
\hline Dialysis & $\begin{array}{l}\text { ICD-9-CM diagnosis codes: V56, V45.1x, 996.73, 458.21; } \\
\text { ICD-9-CM procedure codes: 38.95, 39.27, 39.42, 39.43, 39.95, 54.98, E8791, E8702, E8712, E8722, E8742; } \\
\text { HCPCS codes: A4690, E1510, E1590, E1592, E1594, E1630, E1632, G0257, G0308, G0309, G0310, G0311, G0312, G0313, G0314, } \\
\text { G0315, G0316, G0317, G0318, G0319, G0320, G0321, G0322, G0323, G0324, G0325, G0326, G0327, S9335, S9339; } \\
\text { CPT codes: 90918, 90919, 90920, 90921, 90922, 90923,90924,90925, 90935, 90937, 90945, 90947, 90951, 90952, } 90953,90954, \\
\text { 90955, 90956, 90957, 90958, 90959, 90960, 90961, 90962, 90963, 90964, 90965, 90966, 90967, 90968, 90969, 90970, } 90993, \\
\text { 90997, } 90999,93990,99512\end{array}$ \\
\hline Kidney transplant & $\begin{array}{l}\text { ICD-9-CM diagnosis codes: } V 42.0,996.56,996.68,996.81 \\
\text { ICD-9-CM procedure code: } 55.6 x\end{array}$ \\
\hline
\end{tabular}




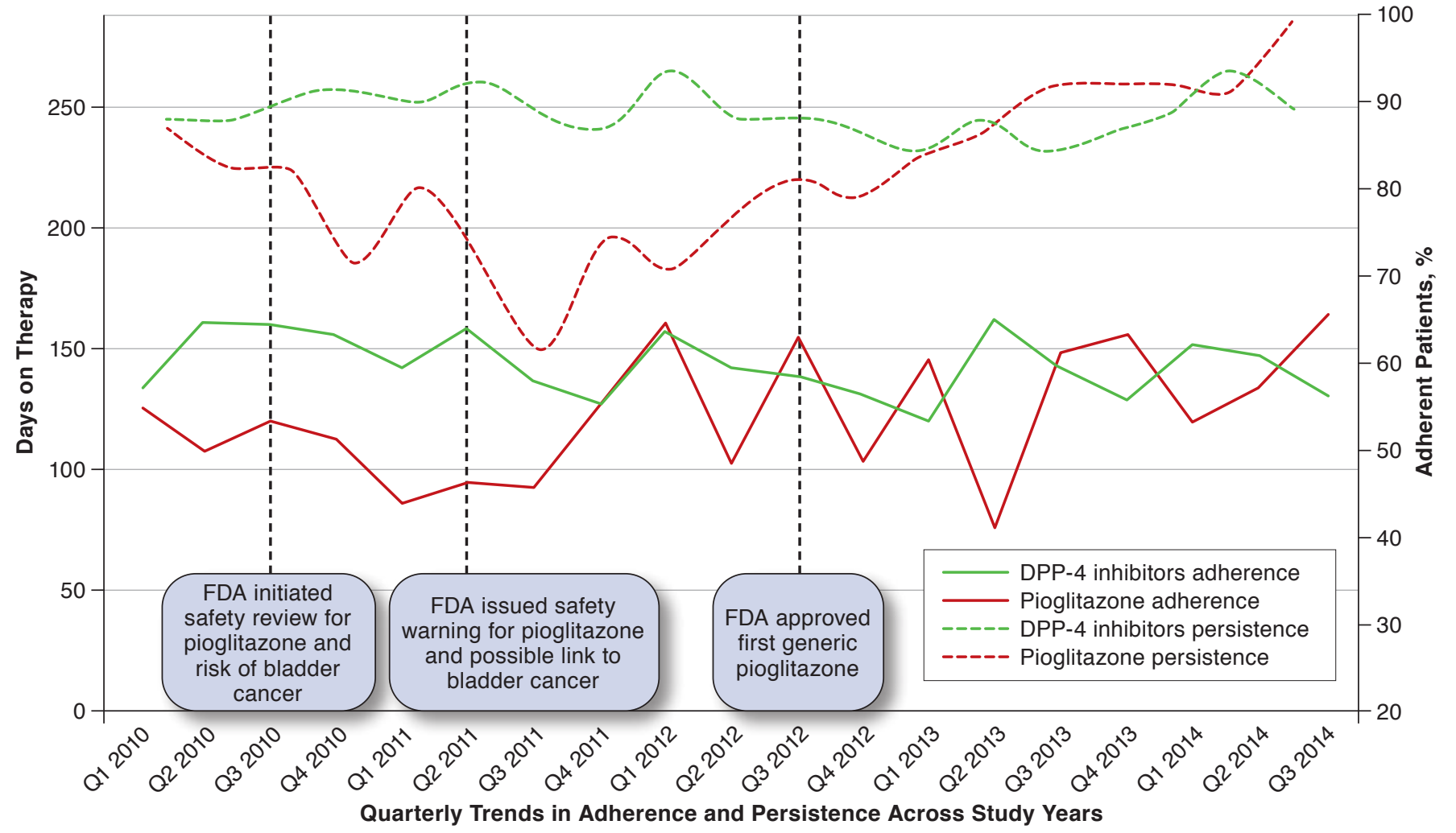

